\title{
The Senile Brucellosis Spondylitis Clinical Diagnosis and Treatment on as Evaluation Were Retrospectively Analyzed
}

\author{
Yao Yao ${ }^{1}$, Xinming Yang ${ }^{*}$ \\ ${ }^{1}$ Hebei North University, Zhangjiakou, China \\ ${ }^{2}$ Department of Orthopaedics, The First Affiliated Hospital of Hebei North University, Zhangjiakou, China \\ Email: *yxm11200@126.com
}

How to cite this paper: Yao, Y. and Yang, X.M. (2018) The Senile Brucellosis Spondylitis Clinical Diagnosis and Treatment on as Evaluation Were Retrospectively Analyzed. Surgical Science, 9, 262-280. https://doi.org/10.4236/ss.2018.98032

Received: March 20, 2018

Accepted: August 21, 2018

Published: August 24, 2018

Copyright $\odot 2018$ by authors and Scientific Research Publishing Inc. This work is licensed under the Creative Commons Attribution International License (CC BY 4.0).

http://creativecommons.org/licenses/by/4.0/ (c) (i) Open Access

\begin{abstract}
Objective: To explore senile brucellosis spondylitis clinical features and diagnostic criteria, in order to improve the diagnosis rate and evaluate the clinical effects of treatment strategies. Methods: From January 2002 to August 2015, 62 patients with Brucella-associated spondylitis were treated with comprehensive diagnosis based on epidemiological history, clinical manifestations, imaging findings, laboratory tests, and local biopsy. The positive rate of red plate agglutination test (RBPT) was $45.1 \%$, the positive rate of serum tube agglutination test (SAT) was $51.6 \%$, and the positive rate of brucellosis anti-human immunoglobulin test (Coomb's) was $100 \%$. All patients underwent X-ray, CT and MRI examinations. The positive rate of blood culture in pathogenic examination was $16.1 \%$, and the positive rate of bone marrow culture was $38.5 \%$. The positive rate of inflammatory granuloma or abscess in the lesion sampling culture was $73.6 \%$. All patients underwent standardized drug therapy and hyperbaric oxygen adjuvant therapy. Among them, 38 patients with neurological impairment were treated with one-stage debridement and posterior pedicle screw fixation on the basis of drug therapy. The paraspinal abscess and the diseased intervertebral space tissue were taken for pathological examination during the operation. One week, two weeks, one month, three months, six months and 12 months after treatment, the patients were evaluated and followed up at the monitoring sites. The evaluation indicators were pain scores, activity of daily living (ADL), imaging findings, and laboratory tests. The SPSS15.0 statistical package was used for analysis. Results: All patients were confirmed by the above-mentioned comprehensive examination after admission. 24 patients (38.71\%) were treated with standard drug therapy (group A), no adverse drug reactions and abnormal liver and kidney function; the remaining 38 patients (61.29\%) were combined with va-
\end{abstract}


rying degrees of neurological impairment, and surgical treatment was performed after 2 to 4 weeks of drug therapy without improvement (group B). The pain scores showed that there was a statistically significant difference between the two groups at the same time point $(\mathrm{P}<0.05)$. The $\mathrm{B}$ group was superior to the A group in both treatment time and pain relief. The blind test evaluation of imaging observation showed that in group B, except for the slow repair of the lesion area, the spine was stable without abscess, sequestrum and inflammatory granulomas, and the score area was stable at $4-5$ points; in group A, after 3 months of treatment, infections in the vertebral body and intervertebral space were not significantly absorbed, and the infection gradually absorbed after 6 months. Laboratory tests showed that over time, the SAT titer, CRP turnover rate, and RBPT negative rate in group B were significantly better than those in group A, indicating that treatment in group B could reduce SAT titer, CRP, and negative RBPT earlier. The clinical curative effect evaluation of 12 months showed that the clinical cure rate in group $\mathrm{B}$ and group A were statistically significant $(\mathrm{P}<0.05)$, indicating that the two groups of treatment methods all had better results, and group $\mathrm{B}$ was better than group A. Conclusion: The senile Brucellosis spondylitis has a characteristic performance. The development of diagnostic criteria can help to improve the diagnostic rate. Standardized drug treatment has a good cure rate. The implementation of surgery has obvious advantages whether it is to relieve pain, stabilize the spine, restore nerve function, or recover early.

\section{Keywords}

Brucelliasis, Spondylitis, Diagnosis, Treatment Strategies, Clinical Evaluation

\section{Introduction}

Brucellar Spondylitis (BS) is an infectious spondylitis caused by a zoonotic, infectious brucella invading the spine. Due to the insensitivity of the body's reaction ability in the elderly, the early clinical symptoms are not typical, and the imaging findings are easily confused with spinal tuberculosis. It is easy to cause misdiagnosis and mistreatment. This disease is an important endemic disease that leads to severely limited spine function in the elderly [1] [2]. Familiar with the clinical features of BS and the diagnosis and treatment plan can play a positive role in the prevention and treatment of the disease. The author retrospectively analyzed 62 cases of elderly BS patients with integrated clinical and follow-up data from January 2002 to August 2015 in our hospital. Through the implementation of systematic diagnosis and treatment program and evaluation of curative effect, good results were obtained. Report as follows.

\section{Methods}

\subsection{Design}

To explore senile brucellosis spondylitis clinical features and diagnostic criteria, 
in order to improve the diagnosis rate and evaluate the clinical effects of treatment strategies.

\subsection{Date and Study Site}

Research done in the First Affiliated Hospital of Hebei North University, Zhangjiakou City, Hebei Province, China from 2002-01 to 2015-08.

\subsection{General Information}

62 elderly patients with BS who were diagnosed in the First Affiliated Hospital of Hebei North University from January 2002 to August 2015, were selected as research subjects. According to the literature [3], BS non-surgical and surgical indications are divided into two groups, non-surgical treatment group 24 cases (A group) to implement standardized non-surgical treatment of drugs, including 17 males and 7 females, aged 65 to 81 years old, the average age was 70.1 years, and the onset time was 4 to 11 months, with an average of 7.5 months. 38 cases were treated with surgery (group B), 27 males and 11 females; aged 65 to 82 years old, average 70.5 years, onset time 5 to 12 Months, an average of 8.5 months. All patients had contact history of cattle and sheep or eating unsterilized dairy products, beef or mutton, among which 15 patients lived in epidemic areas. The main clinical symptoms of the patient were persistent severe low back pain, local tenderness, severe percussion pain, muscle spasm, limited spinal activity, and not daring to walk or turn over, often in a fixed position, but no kyphosis, 14 cases with afternoon remittent fever $\left(\mathrm{T} \leq 38.5^{\circ} \mathrm{C}\right)$, night sweats. All patients were misdiagnosed as other diseases before admission, 58 of which were misdiagnosed as spinal tuberculosis. Lesion vertebrae: 2 vertebrae involved in 56 cases, including L3/L4: 11 cases, L4/L5: 32 cases, L5/S1:13 cases; 3 vertebrae involved 6 cases: L3 L5: 5 cases, L4/L5/S1:1 cases. This group of 38 patients (group B) with different degrees of compression of the cauda equina or nerve roots showed lower limb sensation, movement, reflexes or anal sphincter dysfunction, including 38 cases of sciatic nerve tension signs, 21 cases of compression of the cauda equina. In this group of patients, systemic symptoms improved after drug treatment but local symptoms did not improve and surgery was performed. According to the visual analogue scales (VAS range 0 to 10 points, 0 for no pain, 10 for severe pain), 62 patients had a VAS score of 7 to 10 points on admission.

This study has been approved by the Ethics Committee of the First Affiliated Hospital of Hebei North College. All patients and their families were informed before the medical examination and treatment, and an informed consent form was signed.

\subsection{Laboratory Methods}

In this group of patients, 22 patients (35.4\%) had normal blood routines, and the total number of white blood cells and neutrophilia increased in 6 patients (9.6\%); the total number of white blood cells decreased and lymphocytes in- 
creased in 34 patients (54.8\%); hemoglobin decreased in 42 patients (67.7\%). All patients had a faster ESR $(25-55 \mathrm{~mm} / \mathrm{h})$ and increased CRP $(45-95 \mathrm{mg} / \mathrm{L})$. The rosebengal plate agglutination test (RBPT) was positive in 28 cases $(45.1 \%)$ and negative in 34 cases (54.9\%). The serum tube agglutination test (SAT) titer was more than 1:100 in 32 cases (51.6\%), 30 cases (48.4\%) were less than 1:100. The latter 30 patients were retested for brucellosis anti-human immunoglobulin test (Coomb, s), with a titer of 1:400 or more. All patients had Brucella bacteriological examination + drug susceptibility during fever in hospital, 10 cases (16.1\%) were positive for blood culture, and 52 cases with negative blood cultures received bone marrow culture, of which 20 cases were positive (38.5\%). In 38 patients, inflammatory granulomas or abscesses in the lesions were cultured during surgery and 28 cases were positive (73.6\%). Brucella G-stained microscopic forms of fine Gram-negative tubercle bacilli, no spores, decidua and flagella, no movement, aerobic, contact enzyme-positive, sugar fermentation, the methyl red test, the Viperi test, and the use of cesium and citrate were all negative (Figure 1), sensitive to doxycycline, rifampicin, sulfamethoxazole, streptomycin, penicillin, insensitive to isoniazid, ethambutol, pyrazinamide, amikacin. All patients had negative tumor markers, bone-derived alkaline phosphatase, acidic alkaline phosphatase, anti-hemolytic streptococci O, RF, and HLA-B27.

\subsection{Imaging Methods}

All patients underwent X-ray, CT and MRI examinations. 49 cases (79.1\%) of $\mathrm{X}$-ray showed stenosis of the intervertebral space, increased density, and jagged bone destruction at the edge of the vertebral body (Figure 2), and 13 cases (20.9\%) of vertebral margin hyperosteogeny were beaklike. Bone bridges were formed on the edges of adjacent vertebrae, and lumbar instability was found in 9 cases. 45 cases (72.5\%) of CT showed multiple lesions ranging in size from 0.2 to $0.6 \mathrm{~cm}$ around the edge of the vertebral body. The bone surrounding the lesion

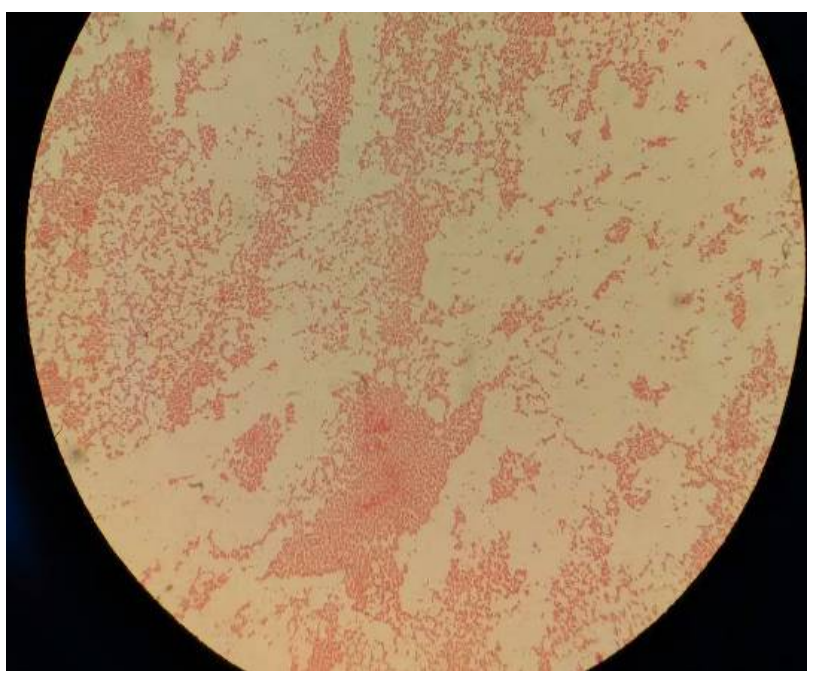

Figure 1. Brucella culture positive. Brucella G-stain shows gram-negative bacillus (oil immersion lens $10 \times 100$ ). 


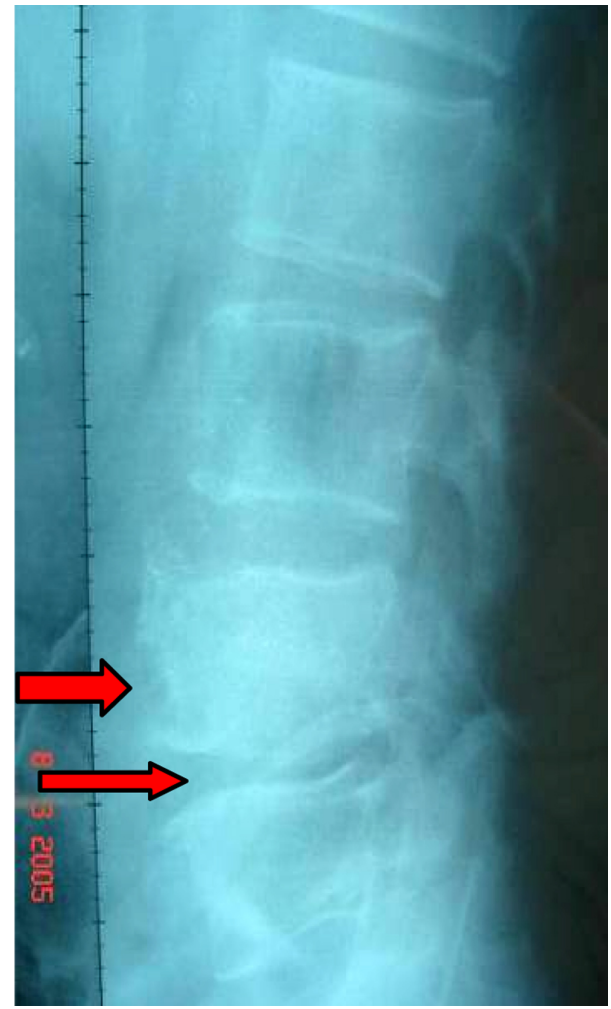

Figure 2. L3-5 Brucellar Spondylitis. X-ray showed stenosis of the intervertebral space, increased density, and jagged bone destruction at the edge of the vertebral body.

was hyperplastic and hardened, and there was a destructive lesion in the newly formed bone tissue to form a "lace vertebra (Figure 3)". In 17 cases (27.5\%), the lesioned intervertebral disc showed Isodensity images. Articular surface hyperplasia, hyperplastic Osteophyte protruding to the edge of the vertebral body to form "lip" osteophyte, The paravertebral and anterior longitudinal ligament form calcification with bone bridge formation (Figure 4). In 10 cases (16.1\%), the psoas major muscles on both sides of the lesioned vertebral plane were widened, abscesses formed inside, and the surrounding fat space was clear, but there was no direct sign of abscess flow (Figure 5). MRI showed uneven signal in 62 cases of vertebral bodies and intervertebral discs. Among them, 10 cases of abnormal signal of thin and unevenly enhanced abscess wall and unclear soft tissue were found near the spine, low signal on T1WI, and high signal on T2WI; 9 cases of lumbar instability with spondylolisthesis; 38 cases of short time inversion recovrey (STIR) showed uneven high signal in the vertebral body, intervertebral discs, attachments, and spinal canal, corresponding to flat dural or nerve root compression (Figure 6), T2WI showed high signal when bone destruction was evident.

\subsection{Pathology Examination}

38 surgical patients, and 17 patients with negative RBPT and normal SAT were 


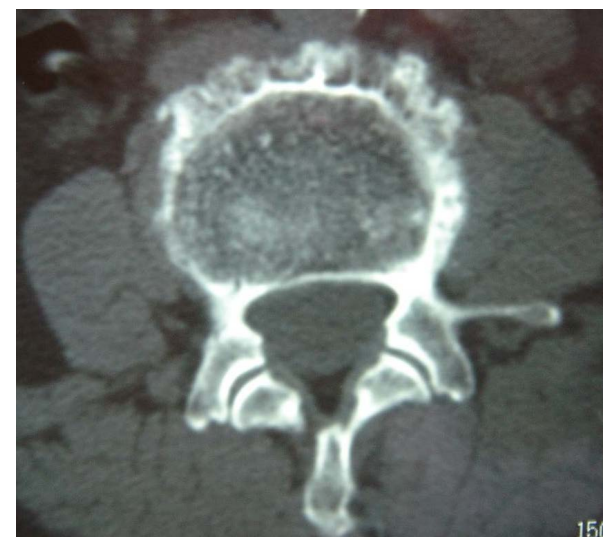

Figure 3. L4 Brucellar Spondylitis. CT showed that the newly formed osteophytes at the margin of the vertebral body and the destruction lesions among them constitute the characteristic performance of "Lace Vertebra".

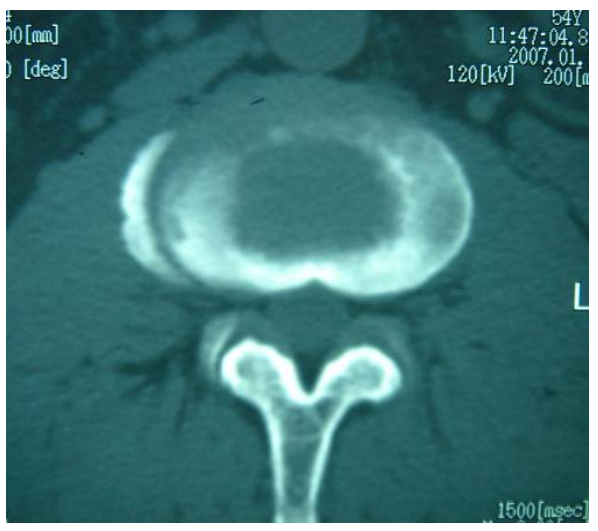

Figure 4. L4 Brucellar Spondylitis. CT showed periosteum hyperplasia, hypertrophy, calcification at the edge of the vertebral body, forming a "lip-like" osteophyte.

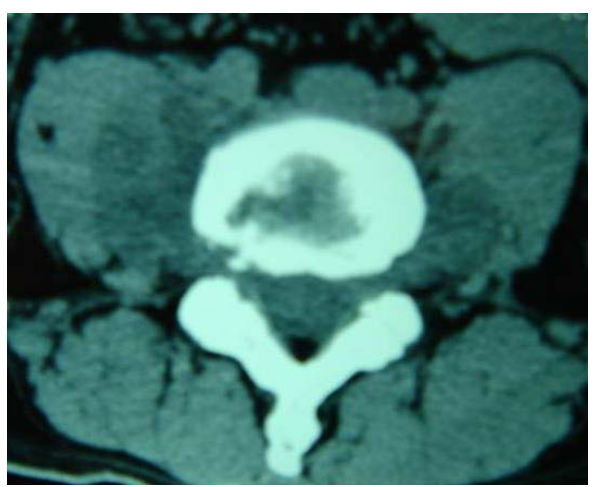

Figure 5. L4 Brucellar Spondylitis. CT showed that the psoas muscles on both sides of the vertebral body were widened, and abscesses formed inside, but not obvious, and the surrounding fat space was clear. 


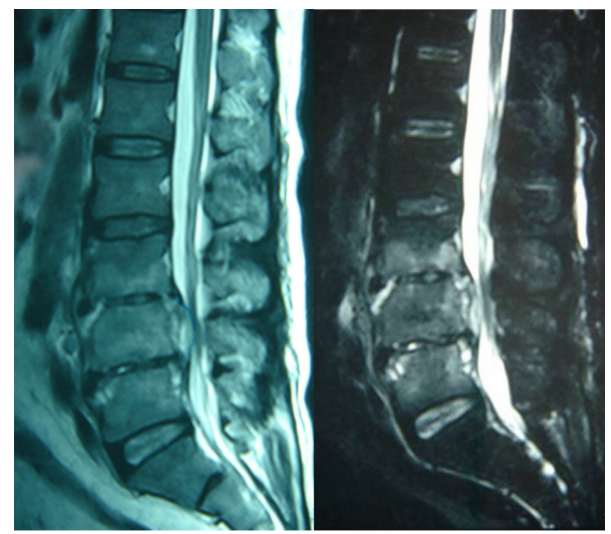

Figure 6. L3-L5 Brucellar Spondylitis. MRI showed thin and unevenly enhanced abscess wall and unclear soft tissue were found near the spine, low signal on T1WI, and high signal on T2WI. STIR showed uneven high signal in the vertebral body, intervertebral discs, attachments, and spinal canal, corresponding to flat dural or nerve root compression.

subjected to acupuncture biopsy under the guidance of C-arm X-ray (8 cases) or CT ( 9 cases). The paraspinal abscess area and the diseased intervertebral tissue were selected for pathological examination. Microscopically, the lesions were seen to have hyperplasia, proliferative nodules, and granuloma formation in the lesion area. There were a large number of mononuclear cells, lymphocytes, neutrophils, and eosinophils infiltrated. And nodular lesions formed of epithelial cells were observed (Figure 7), consistent with the histological presentation of brucellosis.

\section{Treatment Methods}

\subsection{Drug Treatment}

All patients had waist protection during drug treatment, encouraging bed rest and reduced activity. Use long-term, adequate, combined, regular, full-course multi-modal administration. Intravenous infusion of penicillin antibiotics with strong penetrating power to intervertebral disc tissue for $7 \mathrm{~d}$, oral doxycycline 0.1 g bid, first dose doubled, oral 56d, rifampicin $0.45 \mathrm{~g}$, qd, oral 56d, SMZ/TMP 1.0 $\mathrm{g}$ bid, first dose doubled, oral $56 \mathrm{~d}$, the above is a course of treatment, a total of $2-3$ courses of treatment, treatment interval 14d [3] [4].

\subsection{Surgery}

All 38 patients were treated with one-stage debridement and posterior pedicle screw fixation on the basis of drug treatment. The specific surgical method is to first carry out posterior internal fixation, and then to remove the lesion, intervertebral or intertransverse bone grafting. The patient took a prone position, general anesthesia, midline posterior spine incision, conventional exposed 


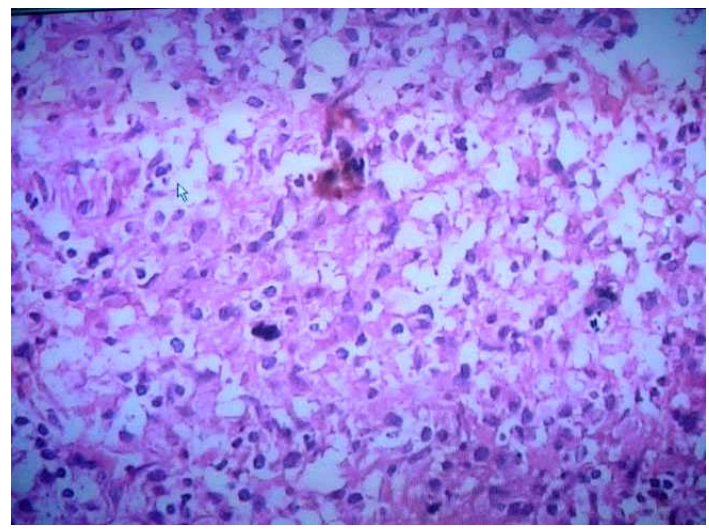

Figure 7. BS under the microscope $\left(\mathrm{HE}^{\star} 200\right)$ showed that the lesions were seen to have hyperplasia, proliferative nodules, and granuloma formation in the lesion area. There were a large number of mononuclear cells, lymphocytes, neutrophils, and eosinophils infiltrated. And nodular lesions formed of epithelial cells were observed.

vertebral spinous process, lamina, articular process, and a vertebra above and below the lesion. The pedicle screws were accurately placed after positioning with a C-arm X-ray machine, If the vertebral body is lightly damaged, less sclerotic bone, and the pedicle and vertebral body are better in bone quality, the pedicle screw can be implanted into the diseased vertebral body after smearing streptomycin, If the damage of the vertebral body is severe or the spine is unstable, one vertebral pedicle screw fixation is performed above and below the lesion. The diseased vertebral lamina was removed, the dural sac and nerve roots of the diseased segment were exposed, and the pus in the spinal canal was removed, there were limited and fusiform inflammatory granuloma or abscess in front of the dural sac or under the posterior longitudinal ligament or around the nerve root. The envelope was incised to scrape off inflammatory granulomas or abscesses and the bone destruction area of the vertebral body was scraped. Then the annulus fibrosus between the two diseased vertebral bodies was incised to scrape the infected disc tissue or destroyed cartilage plate, and the hardened vertebral bone was removed until the bone surface was oozing blood, Under the guidance of a C-arm X-ray machine, the abscess or inflammatory granulomatous tissue in front of the vertebral body (i.e., posterior anterior longitudinal ligament) and the destruction of the anterior vertebral bone were cautiously scraped off with various curettes of various angles. After compression hemostasia and washing repeatedly with hydrogen peroxide, diluted povidone-iodine, normal saline, and gentamicin, the Foraminal mirror was inserted into the intervertebral space to observe the removal of diseased tissue at the anterior longitudinal ligament, and the lesion was removed again when necessary. The diseased articular process and transverse process were polished. The excised lamina crushed and mixed with $1 \mathrm{~g}$ of streptomycin was to implant between the articular process and transverse process. If the amount of bone was insufficient, the autogenous iliac 
bone was taken. Then the drainage tube was placed in the lesion area and the incision was closed. The diseased tissue was taken to do pathological examination. After the operation, the patient rested in bed. After 4 weeks, they used a brace to sit up or get out of bed. Within 3 months, they took the principle of "multiple bed and less activity". Standardized drug treatment was continued after the operation.

\subsection{Curative Effect Evaluation Method}

The patients in this group were evaluated and followed up before and after treatment for 1 week, 2 weeks, 1 month, 3 months, 6 months, and 12 months. The evaluation indicators were pain score, activity of daily living, imaging evaluation, and laboratory test evaluation. Pain was assessed using a visual analogue scales (VAS) scale ranging from 0 to 10 , with 0 indicating no pain and 10 indicating severe pain. Assessment of daily life ability includes: activity level, whether to wear a Thoraco-lumbo-sacral orthoses, when sitting up or getting out of bed, the patient's tolerance for walking time, the ability to go up and down the stairs, ability to stand up from a seat, and whether to return non-manual labor ability before surgery during the first 3 months. The imaging evaluation was to observe whether the spine was stable by X-ray; whether the abscess was disappeared or calcified, whether the contour of the lesion edge was clear and whether the bone destruction was repaired by CT; MRI examinations were performed 3 months, 6 months, and 12 months after treatment (or after surgery). Quantitative evaluation of blind test evaluation indicators for MRI observation was performed. The lesion area was well repaired (infiltration of vertebral inflammation and infection of the intervertebral space were absorbed), spinal stability, no abscess, no sequestrum, no inflammatory granuloma were each 1 point, a total of 5 points, the opposite was 0 points [5]. Laboratory evaluations were performed 3 months, 6 months, and 12 months after treatment (or after surgery) to see if SAT titers, CRP decreased, and whether RBPT was negative. After the treatment (or after surgery) 3 months, 6 months, and 12 months, clinical curative effects were evaluated, and clinical curative effects were expressed as "cured", "improved", and "invalid". The curative effect evaluation standard [6] 1) Cured: The body temperature returns to normal, with complete relief of lumbar pain, full recovery of daily activities, MRI shows of vertebral inflammation and intervertebral space infection has been absorbed, or CT shows abscess disappeared or calcified, the contour of the lesion edge is clear, bone destruction has been repaired, X-ray shows the spinal is stable; SAT titre $<1: 80$ or RBPT negative, CRP $<$ $5 \mathrm{mg} / \mathrm{L}$. 2) improved: The body temperature returns to normal, with relief of lumbar pain $\geq 50 \%$, the recovery of daily activities $\geq 50 \%$, MRI shows of vertebral inflammation and intervertebral space infection has been partially absorbed, or CT shows abscess reduced or calcified, the contour of the lesion edge is relatively clear, bone destruction shows the phenomenon repair, X-ray shows the spinal is stable or not; SAT titers and CRP are lower or RBPT is negative than before. 3) invalid: The body temperature returns to normal or not, with relief of 
lumbar pain $<50 \%$, the recovery of daily activities $<50 \%$, MRI shows no significant change or improvement in vertebral inflammation and intervertebral space infection before treatment., or CT shows no improvement or relapse before treatment, X-ray shows the spinal is stable or not; SAT titers, RBPT, and CRP did not improve before treatment, or RBPT was negative or positive; or there is a short period of improvement in symptoms after treatment, but relapse after 2 weeks of withdrawal.

\subsection{Statistical Methods}

SPSS15.0 statistical software package was used for analysis. Measured data are expressed as mean \pm standard deviation. Multiple groups were compared by repeated measures analysis of variance. If there were statistical differences, $q$ tests were used for multiple comparisons at each time point after treatment and before treatment. Count data were tested by X2. Test level value is 0.05 . The main purpose of the study was to eliminate inflammatory lesions, restore damage to the cauda equina or nerve roots, relieve severe back pain, stabilize spinal structures, and ensure daily activities.

\section{Results}

\subsection{Follow-Up}

All patients were misdiagnosed as other diseases before admission, of which 58 cases were misdiagnosed as spinal tuberculosis, all patients were diagnosed after epidemiological investigation, clinical manifestations, imaging examinations, laboratory tests or pathological examinations. 24 patients in this group were treated with standardized drugs, no adverse drug reactions and liver and kidney function impairment occurred; the remaining 38 patients were combined with different degrees of neurological function injury, and the symptoms were not improved after 2 - 4 weeks of drug treatment, then the surgical treatment was performed. All incisions were primary healing, no complications occurred in the early stage, no recurrence and sinus formation occurred in the late stage, no cases of broken screws or broken rods were observed. After 12 months of follow-up, the intervertebral space or the transverse process and/or articular process bone grafts were all healed. The healing time was $5-10$ months postoperatively with an average of 8 months and the spine was stable. 2 courses of standardized medication was performed after surgery. All patients were evaluated and followed up at 1 week, 2 weeks, 1 month, 3 months, 6 months, and 12 months after treatment (or postoperative).

\subsection{Pain Score Results before and after the Treatment}

The pain scores before and after treatment in group A and B are shown in Table 1. In the two groups, pain scores gradually decreased over time. Scores at each time point in Group B were significantly different from those before treatment $(P<0.05)$, while those in Group A were no difference compared with those 
Table 1. Group A and group B pain index score comparison before and after the treatment $(\mathrm{n}=62, \bar{x} \pm S)$.

\begin{tabular}{|c|c|c|c|c|c|c|c|}
\hline & $\begin{array}{l}\text { Before the } \\
\text { treatment }\end{array}$ & 1 week & 2 weeks & 1 month & 3 months & 6 months & 12 months \\
\hline $\begin{array}{c}\text { Group A } \\
\text { (cases) }\end{array}$ & 24 & 24 & 24 & 24 & 24 & 24 & 24 \\
\hline Pain score & $8.5 \pm 0.7$ & $8.4 \pm 0.6$ & $8.3 \pm 0.6$ & $7.9 \pm 0.5$ & $6.7 \pm 0.4$ & $3.8 \pm 0.3$ & $1.5 \pm 0.2$ \\
\hline$P_{A}$ value & & $>0.05$ & $>0.05$ & $>0.05$ & $<0.001$ & $<0.001$ & $<0.001$ \\
\hline $\begin{array}{l}\text { Group B } \\
\text { (cases) }\end{array}$ & 38 & 38 & 38 & 38 & 38 & 38 & 38 \\
\hline Pain score & $9.2 \pm 0.3$ & $4.6 \pm 0.5$ & $2.1 \pm 0.2$ & $0.7 \pm 0.4$ & $0.2 \pm 0.1$ & 0 & 0 \\
\hline$P_{B}$ value & & $<0.001$ & $<0.001$ & $<0.001$ & $<0.001$ & $<0.001$ & $<0.001$ \\
\hline$P$ value & $>0.05$ & $<0.05$ & $<0.05$ & $<0.05$ & $<0.05$ & $<0.05$ & $<0.05$ \\
\hline
\end{tabular}

$P_{A}, P_{B}$ respectively with than before treatment. $\mathrm{P}$ for group $\mathrm{B}$ compared with group A.

before treatment $(\mathrm{P}>0.05)$, but there was a significant difference at the 3rd, 6th, and 12th months than before $(\mathrm{P}<0.05)$. There was a significant difference between group $B$ and group $A$ at the same time point $(P<0.05)$.

\subsection{Blind Test Evaluation of Imaging Observation}

The scores of blind test evaluation indicators before and after treatment in group A and group B are shown in Table 2. In group A, there were vertebral inflammatory infiltration and intervertebral space infection before treatment, but no spinal instability, abscess, sequestrum and inflammatory granulomas existed. The score was 4 points, and the imaging index score was $4 \pm 0$. After 3 months of treatment, vertebral and intervertebral space infection were not significantly absorbed, and there was no significant difference compared with before treatment ( $P>0.05)$, but after 6 months, the vertebral inflammation and intervertebral space infection were gradually absorbed, which was significantly different from that before treatment $(P<0.05)$. In group $B$, vertebral inflammation, spinal instability, abscess, sequestrum and inflammatory granulomas existed before treatment. The score range was $0-2$, and the imaging index score was $0.17 \pm$ 0.03 on average. After 3, 6 and 12 months of treatment, the spinal stability, no abscess, sequestrum and inflammatory granulomas except the slow repair of the vertebral lesion area, the score was stable at $4-5$, imaging index scores gradually increased, and there was a significant difference at each time point than pretreatment $(\mathrm{P}<0.05)$.

\subsection{Laboratory Testing Index Evaluation}

Laboratory evaluations were performed at the 3rd, 6th and 12th months after treatment (or postoperatively). In group A of 24 patients had a SAT titer (normal value $0-1: 100$ ) of 1:100 or more, 24 cases of CRP (normal reference value $\leq$ $10 \mathrm{mg} / \mathrm{L}$ ) > $40 \mathrm{mg} / \mathrm{L}$, and 13 cases of RBPT (normal negative) positive before 
Table 2. Imaging observation blind test evaluation index score before and after the treatment $(\mathrm{n}=38, \bar{x} \pm S)$.

\begin{tabular}{ccccc}
\hline & $\begin{array}{c}\text { Before the } \\
\text { treatment }\end{array}$ & 3 months & 6 months & 12 months \\
\hline $\begin{array}{c}\text { Group A (cases) } \\
\text { Imaging index } \\
\text { score }\end{array}$ & 24 & 24 & 24 & 24 \\
$\begin{array}{c}\text { (Score area) } \\
P_{A} \text { value }\end{array}$ & $(4-4)$ & $(4-4)$ & $(4-5)$ & $4.75 \pm 0.05$ \\
Group B (cases) & 38 & $>0.05$ & $<0.001$ & $<0.001$ \\
Imaging index & & 38 & 38 & 38 \\
score & $0.17 \pm 0.03$ & $4.68 \pm 0.04$ & $4.92 \pm 0.08$ & $5.0 \pm 0$ \\
(Score area) & $(0-2)$ & $(4-5)$ & $(4-5)$ & $(5-5)$ \\
$P_{B}$ value & & $<0.001$ & $<0.001$ & $<0.001$ \\
\hline
\end{tabular}

$P_{A}, P_{B}$ respectively with than before treatment.

treatment; 8 cases had a SAT titer of 1:100 or more, 8 cases of CRP $>5 \mathrm{mg} / \mathrm{L}, 7$ cases of RBPT positive at the 3rd months; 3 cases had a SAT titer of 1:100 or more, 3 cases of CRP $>5 \mathrm{mg} / \mathrm{L}, 2$ cases of RBPT positive at the 6th months; RBPT was negative, CRP was normal, 2 cases of SAT titer 1:100 at the 12th months. In group B of 38 patients had a SAT titer of 1:100 or more in 20 cases, 38 cases of CRP $>40 \mathrm{mg} / \mathrm{L}$, and 15 cases of RBPT positive before treatment; 11 cases had a SAT titer of 1:100 or more, 9 cases of CRP $>5 \mathrm{mg} / \mathrm{L}, 4$ cases of RBPT positive at the 3rd months; only 1 cases had a SAT titer of 1:100 or more, 1 cases of CRP > $5 \mathrm{mg} / \mathrm{L}$, RBPT was negative in all cases at the 6th months; RBPT was negative, CRP and SAT were normal at the 12th months.

\subsection{Clinical Curative Effect Evaluation}

Clinical efficacy evaluation was performed at the 3rd, 6th, and 12th months after treatment (or postoperative) (Table 3). The difference of the cure rate between the latter and the previous time point in the same group was statistically significant $(\mathrm{P}<0.05)$; the clinical cure rate at the same time point in group $\mathrm{B}$ was better than that in group $\mathrm{A}$, and the difference was statistically significant $(\mathrm{P}<$ $0.05)$.

\section{Discussion}

Brucellosis is a zoonotic infectious endemic disease caused by brucellosis. In recent years, the incidence rate of the elderly has gradually increased. Due to the low physiological function of the elderly, the body has low immunity, slow metabolism, and mild inflammatory response, and most associated with lumbar degeneration. Once infected with brucellosis in the elderly, it is highly prone to a secondary infection of the spine, i.e. BS. Therefore, the clinical manifestations of elderly BS are slow and atypical, drug therapy is not sensitive, long duration, recurrent, long-term unrecoverable, poor efficacy, and high recurrence rate. The 
Table 3. In the two groups after treatment clinical curative effect evaluation at each time point ( $\mathrm{n}=38, \bar{x} \pm S$ ).

\begin{tabular}{|c|c|c|c|c|c|c|c|c|c|c|c|c|c|c|c|c|}
\hline & \multicolumn{6}{|c|}{3 months } & \multicolumn{5}{|c|}{6 months } & \multicolumn{5}{|c|}{12 months } \\
\hline & cases & cure & mprov & nvalid & $\begin{array}{l}\text { cure } \\
\text { rate }\end{array}$ & $\begin{array}{l}\text { improvement } \\
\text { rate }\end{array}$ & cure & mpro & nvalid & $\begin{array}{l}\text { cure } \\
\text { rate }\end{array}$ & $\begin{array}{l}\text { improvement } \\
\text { rate }\end{array}$ & cure & nprove & nvalid & $\begin{array}{l}\text { cure } \\
\text { rate }\end{array}$ & $\begin{array}{l}\text { improvement } \\
\text { rate }\end{array}$ \\
\hline GA & 24 & 4 & 13 & 7 & 16.67 & 70.83 & 16 & 5 & 3 & $66.67^{\Lambda}$ & 87.50 & 21 & 3 & 0 & $87.50^{\Lambda}$ & 100 \\
\hline GB & 38 & 30 & 8 & 0 & $78.95^{\mathbf{V}}$ & 100 & 35 & 3 & 0 & $92.11^{\Delta \boldsymbol{V}}$ & 100 & 38 & 0 & 0 & $100^{\Lambda \boldsymbol{V}}$ & 100 \\
\hline $2 \mathrm{G}$ & 62 & 34 & 21 & 7 & 54.84 & 88.71 & 51 & 8 & 3 & $82.26^{\mathbf{\Lambda}}$ & 95.16 & 59 & 3 & 0 & $95.16^{\mathbf{\Lambda}}$ & 100 \\
\hline
\end{tabular}

Note: the cure rate (cured cases/total number of cases $\times 100 \%$ ) and improvement rate (cure + to improve the number of cases/total number of cases $\times$ $100 \%)$. ${ }^{\star}$ Compared with the former point data of group P $<0.05$; ${ }^{\circ}$ compared to the same point group A P $<0.05$.

clinical manifestations and X-ray examination of BS have many similar manifestations with spinal tuberculosis. It is easy to cause misdiagnosis and mistreatment [1]. 62 cases were misdiagnosed before admission, of which 58 cases were misdiagnosed as spinal tuberculosis. The misdiagnosis rate was $93.54 \%$. However, as long as the clinician recognizes and values the disease, is familiar with the epidemiological characteristics of the disease, and recognizes its clinical imaging, laboratory, and pathological features, it can make a differential diagnosis. Therefore, to deepen the understanding of BS, make clear diagnosis, reduce misdiagnosis, it is of great significance for the definite diagnosis of the disease and the timely and effective standardization of drug treatment and precise surgical intervention.

\subsection{BS Clinical Characteristics and Diagnosis}

In addition to the epidemic history of BS patients, such as having close contact with livestock or livestock products, Brucella culture, etc., or living in epidemics such as epidemic areas, the clinical manifestations of BS patients are divided into general manifestations and local manifestations. Systemic manifestations are brucellosis toxicity symptoms. Patients have headaches, remittent fever, night sweats, fatigue, loss of appetite, and anemia. They are often accompanied by respiratory and reproductive system infections. They may have enlarged liver, spleen, lymph nodes, and testes, as well as multiple, migratory muscles and large joint pain. Local manifestations are infectious spondylitis symptoms, lumbar vertebral invasion is the most common, persistent severe low back pain, local tenderness, percussive pain, muscle spasms, and limited spinal activity, often in a fixed position, can form psoas muscle abscess, with corresponding nerves Root radiating pain or cauda equina compression symptoms [6] [7].

The most commonly used serological methods for laboratory examination for brucellosis are RBPT and SAT. Since the positive rate of RBPT is low $30.6 \%$ in this group), SAT is often used clinically. It is positive after 2 weeks of onset. $80 \%$ of patients in the acute phase are positive for SAT, while $30 \%$ of patients in the chronic phase have positive SAT. SAT is less affected by other factors, even if the course of the disease is longer or has received anti-inflammatory treatment, it may have a higher positive rate, which can be used as the most common method for diagnosis and differential diagnosis. The elderly were not sensitive to body 
reaction and had a long medical history. The positive rate of SAT was 51.6\%. Therefore, SAT-negative patients could not rule out brucellosis. Although the positive rate of Coomb's is high, the positive rate in this group is $100 \%$, but due to the influence of hospital technical equipment and reagents, it cannot be used as a clinical routine. The diagnostic value of Brucella culture in bacteriology is high, but the positive rate of blood culture varies from $10 \%$ to $74 \%$ [8]. The positive rate of blood culture in this group is only $16.1 \%$, the positive rate of bone marrow culture is $38.5 \%$, and focal inflammatory granuloma or abscess culture positive rate was $50.9 \%$. The reason for the low positive rate of blood culture may be the duration of the disease, whether it is in the state of bacteremia, whether or not the systemic infection is combined, whether it is treated with anti-infective treatment before blood collection, etc. $93.5 \%$ of patients in this group were misdiagnosed as spinal tuberculosis before admission and were treated with anti-tuberculosis chemotherapy. Rifampin and streptomycin were also the main drugs for the treatment of brucellosis. This is the main reason for the low positive rate of blood culture. Therefore, clinical bacteriological cultures should be focused on the sampling of lesions or bone marrow culture to increase the positive rate.

The early X-rays are often found without abnormalities, and generally change in the 6 - 8 weeks after the onset. Once X-ray signs appear, it develops rapidly, mainly destroys the vertebral body and narrows the intervertebral space. Bone destruction is concentrated on the upper or lower edge of the vertebral body, manifesting as progressive intervertebral space stenosis and involving two adjacent vertebral bodies. Once X-ray signs appear, it develops rapidly, mainly destroys the vertebral body and narrows the intervertebral space. Bone destruction is concentrated on the upper or lower edge of the vertebral body, manifesting as progressive intervertebral space stenosis and involving two adjacent vertebral bodies, can be associated with the destruction of the facet joint, but no vertebral compression or wedge deformation. Bone bridges appear at the later stage and are extremely hard but rarely bone fusion. 49 cases show intervertebral stenosis, bone destruction, 13 Cases form bone bridges; CT examination can clearly show the location of the lesion, the degree of bone destruction, the presence of cavity and sequestrum, the destruction of the disc is iso-density, and the periosteum of the vertebral is hyperplastic and calcified to form a "lip-shaped" osteophyte. New destructive lesions appear in the newly formed bone at the edge of the vertebra, which constitutes a "lace vertebral". Less abscess around the vertebral body, no sequestrum and pedicle destruction. "Lace vertebrae" and "lip" Osteophyte are characteristic features of CT. In particular, it has unique advantages in displaying abscess around the lesion. They can also be used for puncture and biopsy under CT guidance. In this group, 45 cases had "lacquer vertebrae" and 17 cases had "lip" osteophytes, 10 cases showed paravertebral abscesses; the main value of MRI examination is to guide surgery. It can show abnormal signals in the inflammatory infiltration stage, locate the affected segment and the 
extent of lesions, clearly show the inflammatory vertebral body, damaged intervertebral discs, paravertebral abscesses, and intraspinal and epidural abscesses, corresponding flat dural sac compression, which is caused by disrupted intervertebral disc or inflammatory granulation tissue protruding into the spinal canal [2] [6] [7]. 38 cases of MRI showed dural sac compression.

Pathology is characterized by hyperplasia. Proliferative nodules and non-tuberculous granuloma are formed in the lesion with mononuclear cells, lymphocytes, neutrophils, and eosinophils infiltrating, and nodular lesions composed of epithelial cells can be seen [1] [6]. In group B, the pathological examination of the lesions removed during operation was consistent with the pathology of brucellosis.

There are no uniform diagnostic criteria for BS. In particular, the clinical symptoms of the elderly are not typical. The diagnosis of this disease should be combined with epidemiological history, clinical manifestations, imaging findings, laboratory examination, and local biopsy to perform comprehensive diagnosis [9] [10]. For suspected cases, immunology, pathology, and etiological examination should be actively conducted to make clinically diagnosed cases become confirmed cases [11]. 62 elderly patients met BS confirmed cases.

\subsection{BS Treatment Strategies}

BS is an intervertebral discitis or vertebral body inflammation caused by brucellosis invading the spine. Domestic and foreign treatment methods are still based on drug therapy. Most patients can be cured by conservative treatment [3] [8] [12]. According to the principles laid down in the WHO's sixth joint communique, tetracycline + streptomycin, or similar drugs based on this regimen were used for treatment. Many scholars at home and abroad observed that the efficiency of this method is not very high at only $60 \%$. No matter what kind of drug for the treatment of brucellosis, its long-term efficacy is poor, and the recurrence rate is indeed high [4] [13] [14]. By re-evaluating the effect of drug compatibility and treatment on the curative effect of Brucella-associated spondylitis, the current ideal plan is: Doxycycline $0.1 \mathrm{~g}$, bid, for the first time doubled, for $56 \mathrm{~d}$; rifampicin $0.6 \mathrm{~g}$, qd, 56d; sulfamethoxazole $1.0 \mathrm{~g}$, bi d, for 56d; using doxycycline + rifampin + sulfamethoxazole as the first choice, meeting Sanford's preferred medication in the Guide to Antimicrobial Therapy [15] [16]; reported in the literature that the cure rate for BS is about $85 \%$, and about $95 \%$ efficiency [4]. Ioannou confirmed through research that no matter which treatment plan is adopted, the longer-term treatment plan is positively correlated with the lower failure rate, which is consistent with the results reported by Seyed [16] [17]. 38 patients in group B were treated with this therapy for two courses before and after surgery, and administered at 7-day intervals. After RBP was converted to negative, medication was continued for two weeks. The cure rate of this therapy was $86.84 \%$, and the improvement rate was $100 \%$. On the basis of standardized drug therapy, surgery is performed on patients with the following symptoms [6] 
[18] [19] [20] [21]: 1) With paravertebral abscess or psoas muscle abscess that is relatively large and difficult to absorb; 2) Intraspinal abscesses Or inflammatory granuloma; 3) Intractable pain caused by intervertebral disc destruction; 4) Larger vertebral damage or destruction of the articular process affects the stability of the spine; 5) Spinal cord, cauda equina or nerve root compression; 6) Mixed with other bacterial infections. All of the patients in Group B were associated with intractable pain caused by varying degrees of cauda equina or nerve root compression, and intervertebral disc destruction. There was no improvement after 2 - 4 weeks of conservative treatment. The imaging showed 9 cases of lumbar instability with spondylolisthesis, and 10 cases of psoas muscle abscess formed on both sides of vertebral lesions, and 38 cases of MRI showed spinal epidural abscess, disrupted disc or inflammatory granulation tissue into the spinal canal, the corresponding flat dural sac, cauda equina or nerve root compression. 38 patients in group B were eligible for surgical indications. The surgical strategy was closely related to the four therapeutic goals of "curing the lesion, reconstructing spinal stability, restoring spinal cord function, and early recovery" [18]. Clinical observations indicate that the recurrence of brucellosis spondylitis is related to the instability of the spine [22]. Relying solely on the removal of the lesion to promote the healing of the infection is no longer the purpose of the surgical treatment [18] [19] [21] [22] [23]; Therefore, 38 patients in group B were treated with one-stage debridement combined with posterior pedicle screw fixation. The operation not only cleared the lesion, relieved the compression of the spinal nerve system, but also restored the stability of the spine and promoted the patient's early recovery. In group A, 24 patients had no indications for surgery. MRI showed only heterogeneous signal in the vertebral body and intervertebral discs, (i.e., inflammatory infiltration of the vertebral body and intervertebral space), no sequestrum and large bone destruction lesions, no paravertebral or intraspinal abscesses, and no disc damage or inflammatory granulation tissue. Standardized drug treatment strategies have achieved good results.

\subsection{BS Curative Effect Evaluation}

Pain index score comparison before and after treatment (Table 1) showed: In group $\mathrm{A}$, there was no difference before and after treatment within 1 month $(\mathrm{P}>$ $0.05)$, and there was a statistically significant difference between the rest of the time and before treatment $(\mathrm{P}<0.05)$. In group $\mathrm{B}$, there was a statistically significant difference between the scores at each time point and before treatment $(\mathrm{P}<$ $0.05)$, indicating that with the passage of time, the two groups of treatment methods can gradually reduce the pain score, i.e., effectively relieve pain. There was a statistically significant difference between group B and group A at the same time point $(\mathrm{P}<0.05)$, indicating that in group $B$, the surgical method of reconstructing the stability of the spine based on the removal of the lesions can effectively relieve the severe pain caused by Infection, cauda equina or nerve root 
compression, and instability of the spine. The pain can be completely relieved after 3 months. However, in group A, the pain was gradually relieved after 3 months of pure drug therapy, and the pain of individual patients was not completely relieved at 12 months, indicating that the group B treatment was superior to group A at both time and pain relief.

Imaging observation blind test evaluation index score before and after the treatment (Table 2) showed: in group A, the difference was statistically significant $(\mathrm{P}<0.05)$ after 6 months of treatment compared with that before treatment, indicating that the treatment of group A could also make the inflammatory infiltration of vertebral bodies and intervertebral space infection gradually absorbed, but it took a long time than group B. In group B, except that the early vertebral lesions were not repaired well due to lack of time,, there were no spinal instability, abscesses, sequestrum, and inflammatory granulomas. The scores were stable at 4 - 5 points, and were gradually increased, indicating that operation under direct vision or assisted C-arm X-ray machine and combined with the guidance of an intervertebral foramen, can completely remove the lesion. And pedicle screw fixation can provide the spine instant stability at early stage and lasting stability at later stage. Over time, the vertebral lesions were continuously repaired by self-repair ability or bone grafting. The 12-month follow-up imaging showed that the lesion area was well repaired. And there was a statistically significant difference between each time point and preoperative $(P<0.05)$.

The results of laboratory tests at the same time points of 3 months, 6 months, and 12 months after treatment showed that compared with before treatment, the SAT titer reduction rate, $\mathrm{CRP}$ reduction rate, and $\mathrm{RBPT}$ negative rate in group $\mathrm{B}$ were significantly better than those in group A. It indicated that drug treatment combined with surgery can make SAT titer and CRP lower or RBPT negative earlier than drug treatment alone.

Clinical curative effect evaluation at each time point after treatment (Table 3) showed: Over time, symptoms in both groups gradually improved and healed. In the same group, the difference between the cure rate at the latter time point and the cure rate at the previous time point was statistically significant $(\mathrm{P}<0.05)$, indicating that the two groups of treatment methods had good results for the elderly BS. Compared with the two groups at the same time point, 38 patients in group B had 30 cured patients and 8 patients improved in 3 months, the improvement rate was $100 \%$, and the cure rate was $78.95 \%$. This shows that compared with group $\mathrm{A}$, the implementation of surgery had a significant effect in improving the early efficacy of BS $(\mathrm{P}<0.05)$, and at the subsequent time point, the cure rate of group $B$ was significantly better than that of group A $(\mathrm{P}<0.05)$. It was demonstrated that this surgical procedure had a better cure rate for pain relief, restoration of ADL, and repair of neurological impairment in senile BS patients with surgical indications, both in the early and late stages. Therefore, surgery is extremely beneficial for removing lesions, controlling infections, restoring the function of the cauda equina and nerve roots, reducing the disability 
rate, and increasing the cure rate [21] [24] [25].

\section{Fund}

Key project of technical research and achievement transformation in health field of Hebei province (zh2018014); Hebei medical application technology tracking project in 2018(G2018074); Science and technology program of Zhangjiakou in 2017(17120010D).

\section{Conflicts of Interest}

The authors declare no conflicts of interest regarding the publication of this paper.

\section{References}

[1] Yang, X.-M., Meng, X.-Y., Shi, W., et al. (2014) The Comparison of the Manifestation of the Clinical Imageology and Pathology between the Brucellar Spondylitis and the Spine Turberculosis. Scientific Research, 5, 60-69.

[2] Yang, X.-M., Shi, W., Wang, X.-Y., et al. (2014) Imaging Classification and Clinical Significance of Brucellosis Spondylitis. The Spine Journal, 3, 172-177.

[3] Saltoglu, N., Tasova, Y., Inal, A.S., et al. (2002) Efficacy of Rifampicin Plus Doxycycline Versus Rifampicin Plus Quinolone in the Treatment of Brucellosis. Saudi Medical Journal, 23, 921-924.

[4] Yang, X.-M., Shi, W., Meng, X.-Y., et al. (2013) The Assessment of the Clinical Effect of the Drug Compatibility and Course of Treatment to the Brucellar Spondyliti. Scientific Research, 4, 92-99.

[5] Yang, X.-M., Meng, X.-Y., Hu, C.-B., et al. (2016) The Diagnosis and Therapeutic Value of MRI Images Typing for Brucella Spondylitis Disease. Chin J Anat Clin, 21, 101-108.

[6] Yang, X.-M., Shi, W., Du, Y.-K., et al. (2008) The Clinical Characteristics and Surgical Treatment of Brucellar Spondylitis. Chinese Journal of Orthopedics, 28, 35-40.

[7] Meng, X.-Y., Yang, X.-M., Zhang, P., et al. (2015) Surgical Outcomes of 38 Cases of Brucellar Spondylitis with Nervous Lesion. Orthopedic Journal of China, 23, 393-401.

[8] Turgut, M., Turgut, A.T. and Kosar, U. (2006) Spinal Brucellosis: Turkish Experience Based on 452 Cases Published during the Last Century. Acta Neurochirurgica, 148, 1033-1044. https://doi.org/10.1007/s00701-006-0877-3

[9] Zhang, P., Yang, X.-M. and Meng, X.-Y. (2013) Brucella Spondylitis Diagnosis and Treatment. Chinese Journal of Spine and Spinal Cord, 23, 1029-1032.

[10] Mairdan,-M., Ti, J., Sheng, W.-B., et al. (2012) Diagnosis and Surgical Treatment of the Brucellar Spondylitis. Chinese Journal of Orthopaedics, 32, 323-330.

[11] Zhang, Z., Yang, X.-M., Shi, W., et al. (2014) Diagnosis and Treatment of Lumbar Brucellosis Spondylitis [J/CD]. Chin J Exp Clin Infect Dis (Electronic Edition), 8, 8-13.

[12] Yang, X.-M., Shi, W. and Du, Y.-K. (2008) Investigation on the Curative of Brucellar Spondylitis. Chinese Journal of Endemiology, 27, 699-703.

[13] Solera, J., Geijo, P., Largo, J., et al. (2004) Arandomized, Double-Blind Study to Assess the Optimal Duration of Doxycycline Treatment for Human Brucellosis. Clini- 
cal Infectious Diseases, 39, 1776-1782. https://doi.org/10.1086/426024

[14] Alp, E., Koc, R.K., Durak, A.C., et al. (2006) Doxycycline Plus Streptomycin versus Ciprofloxacin Plus Rifampicin in Spinal Brucellosis [ISRCTN31053647]. BMC Infectious Diseases, 11, 72-74. https://doi.org/10.1186/1471-2334-6-72

[15] Gilbert, D.N., Moellering, R.C., Eliopoulous, G.M. and Sande, M.A. (2004) The Sanford Guided to Antimicrobial Therapy. Antimicrobial Theraphy, 40, 48-51.

[16] Ioannou, S., Karadima, D., Pneumaticos, S., et al. (2011) Efficacy of Prolonged Antimicrobial Chemotherapy for Brucellar Spondylodiskitis. Clinical Microbiology and Infection, 17, 756-762. https://doi.org/10.1111/j.1469-0691.2010.03272.x

[17] Seyed, M., Mohammad, R., Nasser, J., et al. (2012) Outcomes of Treatment in 50 Cases with Spinal Brucellosis in Babol, Northern Iran. The Journal of Infection in Developing Countries, 6, 654-659.

[18] Yang, X., Meng, X. and Zhang, Y. (2012) Treatment of the Brucellar Spondylilis in the Thoracic and Lumbar Vertebrae by Surgery. Chinese Journal of Spine and Spinal Cord, 22, 1055-1058.

[19] Yang, X., Zhang, L., Zhang, Y., et al. (2012) Clinical Evaluation of One-Stage Radical Debridement Combined with Posterior Pedicle Internal Fixation Is an Ideal Therapeutic Method for Brucellar Spondylitis of the Thoracic and Lumbar Vertebrae. Chinese Journal of Reparative and Reconstructive Surgery, 26, 266-271.

[20] Pina, M.A., Modrego, P.J. and Uroz, J.J. (2001) Brucellar Spinal Epidural Abscess of Cervical Location: Report of Four Cases. European Neurology, 45, 249-253. https://doi.org/10.1159/000052137

[21] Atonis, P., Tzermiadianos, M., Gikas, A., et al. (2006) Surgical Treatment of Spinal Brucellosis. Clinical Orthopaedics and Related Research, 444, 66-72. https://doi.org/10.1097/01.blo.0000203455.59393.9a

[22] Yang, X., Zhang, L., Jia, Y., et al. (2015) Retrospective Analysis of Posterior or Anterior Debridement with Internal Fixation for Lumbar Brucellosis Spondylitis. Chinese Journal of Clinical Anatomy, 20, 428-434.

[23] Bouaziz, M.C., Bougamra, I., Kaffel, D., et al. (2010) Noncontiguous Multifocal Spondylitis: An Exceptional Presentation of Spinal Brucellosis. La Tunisie Médicale, 88, 280-284.

[24] Namiduru, M., Karaoglan, I. and Gursoy, S. (2004) Brucellosis of the Spine: Evaluation of the Clinical, Laboratory, and Radiological Findings of 14 Patients. Rheumatology International, 24, 125-129. https://doi.org/10.1007/s00296-003-0339-7

[25] Du, X. and Yang, X. (2015) One-Stage Posterior Lumbar Radical Debridement, Interbody Fusion, and Posterior Short-Segment Pedicle Internal Fixation in Treatment of Lumbar Brucellar Spondylitis. Journal of Practical Orthopaedics, 21, 110-114. 